\title{
International Branch Campuses-Curiosity or Important Trend?
}

\section{RICHARD GARRETT}

Richard Garrett is director, Observatory on Borderless Higher Education. E-mail: richard.garrett@i-graduate.org. The Observatory on Borderless Higher Education (OBHE) has teamed up with the CrossBorder Education Research Team (C-BERT at SUNY Albany and Penn State University) to put together a new report on international branch campuses. Part 1 is available now for Observatory members and can be purchased by nonmembers.

Thternational branch campuses (IBCs) have emerged as a 1 distinctive aspect of the internationalization strategies of governments and higher education institutions. These entities captured a great deal of attention during the 2000 s as institutions rushed to set up shop-particularly in certain Middle Eastern and Asian countries-anticipating some mix of recruitment, revenue, research, and branding gains. Some of these adventures ended in well-publicized failures and others have become very successful. Today, we count 249 branches operating around the world-up from 66 in 20II, with around 20 believed to be in development.

Examples include University of Nottingham's campuses in Malaysia and China, Georgia Tech's campus in France, RMIT's campus in Vietnam, and the Philippines AMA International University campus in Bahrain.

True IBCs are still quite rare but continue to open with some frequency. If we include IBCs that have changed status or closed in the past, of which there are at least 42 documented instances, there have been 29I IBCs created in total.

\section{WHAT IS AN IBC?}

The new report, which was published in November 20I6, defines an IBC as "an entity that is owned, at least in part, by a foreign education provider; operated in the name of the foreign education provider; and provides an entire academic program, substantially on site, leading to a degree awarded by the foreign education provider."

Gathering information about IBCs is difficult, as there is no governmental or nongovernmental entity that officially tracks such activity. Few countries systematically collect information on the foreign activities of their higher education institutions. Attempts were made to gather data from every IBC in existence, through the institutional website, online news articles and press releases, or via e-mail with institutional leaders. Not every institution had data readily available or were willing to share, and some offered incomplete data. More comprehensive and publicly available data would be of great benefit to all stakeholders in IBC ventures. Our data set offers the most comprehensive picture of the IBC landscape to date.

The full Part I report provides a complete list of known IBCs in operation and under development, along with data on year established, degrees and programs offered, and student numbers. It also offers analysis of typologies, government rationales and motivations for opening IBCs, and the various quality assurance models in place.

\section{How Many Students Are Enrolled? Where are IBCS LOCATED?}

The OBHE and C-BERT teams estimate that at the end of 20I5, about I80,000 students worldwide were enrolled in IBCs as defined in this report. This is a significant number in absolute terms, but it is equivalent to less than 4 percent of the five million international students in the world-students who study in another country-and a tiny fraction of the more than 150 million higher education students globally. In a few countries, such as the United Arab Emirates (UAE), IBCs constitute a significant proportion of the total higher education enrollment; but, in most, they are niche players.

\section{International branch campuses (IBCs) have emerged as a distinctive aspect of the internationalization strategies of governments and higher education in- stitutions.}

Overall, there are now 33 "home"-or source-countries for IBCs, up from 28 at the start of 2011 . The top five home countries are the United States, the United Kingdom, Russia, France, and Australia. Together, these countries account for I8I branch campuses, or 73 percent of the world's IBCs. There are now 76 host countries, up from 69 countries at the start of 20II. The top five host countries are China, the UAE, Singapore, Malaysia, and Qatar, which together host 98 IBCs, or 39 percent of the world's total IBCs.

\section{Do IBCS MATTER?}

IBC rationales span revenue, institutional internationalization and two-way mobility, prestige, and securing a base for research. There is little evidence that IBCs generate atypical surplus, and much, if not all, net income is ploughed back 
into the operation. Short-term benefits are few, and, inevitably, it takes many years for an IBC to become established, and to judge its impact.

IBCs are pursued both by elite institutions that see an international campus as a high-status differentiator, and by less well-known institutions that may be freer from tradition and see an international presence as a way to create fresh brand perceptions in new markets.

Institutions that invest in IBCs are playing the long game, betting on a more globalized future where deep international presence is seen to define a university. Today, most IBCs are still reshaping the model, concerned largely with in-country students and seeing little two-way mobility or single-brand enhancement. As has happened in the past, some IBCs may gradually become independent of the parent institution and transform into a domestic university. The added value of an international network of campuses, where the sum is greater than the parts, is still a horizon for institutions engaged with IBCs.

What is certain is that if IBCs do emerge as important indicators of institutional effectiveness and reach, it will be very difficult for other institutions to catch up. A global intercampus network at which all students pursue their studies, or close government and corporate relationships fostered over decades, cannot be replicated overnight. Some universities are banking on smaller international centers as a better balance of risk and reward. Ohio State University's Global Gateways model is a good example.

The Observatory and C-BERT will continue to track the IBC phenomenon. Indeed, Part 2 of the IBC report, to be published in 2017 , will be based on interviews with institutional leaders at a sample of IBCs in operation for at least a decade. It will investigate motivations and operations of mature IBCs, explore the question of how to judge success from different perspectives, and what combination of conditions breeds success.

DOI: http://dx.doi/org/I0.6oI7/ihe.20I7.90.9743

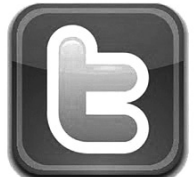

In addition to our Web site and Facebook page, we are now tweeting. We hope you will consider "following" us on Twitter!

\section{Twenty-first Century Mobil- ity: The Role of International Faculty}

\section{Philip G. Altbach and Maria Yudkevich}

Philip G. Altbach is research professor and founding director of the Center for International Higher Education at Boston College, US. E-mail: altbach@bc.edu. Maria Yudkevich is associate professor of economics and vice-rector at the National Research University Higher School of Economics, Moscow, Russia. E-mail: 2yudkevich@gmail.com. This article stems from research done for International Faculty in Higher Education: Comparative Perspectives on Recruitment, Integration, and Impact, edited by M. Yudkevich, P. G. Altbach, and L. E. Rumbley. (Routledge 2017).

$\mathrm{T}$ the era of globalization, it is not surprising that grow1 ing numbers of academics are working outside of their home countries. Universities are themselves increasingly globalized-they are perhaps the most globalized of all prominent institutions in society. Even though the global percentage of international academics is small, this group is quite important. We broadly define international faculty as academics that hold appointments in countries where they were not born and/or where they did not receive their first postsecondary degree. In most cases, they are not citizens of the country in which they hold their academic appointment. They are drivers of international consciousness at universities, they are often top researchers, and, in some countries, they constitute a large percentage of the academic labor force.

International faculty seem to cluster into five broad categories. A small but highly visible group of international faculty hold appointments at top research universities around the world, especially in the major English-speaking countries-Australia, Canada, the United States, and to some extent the United Kingdom. They are the global superstars, and some hold Nobel and other important prizes. A second group is employed by midrange or upper-tier universities in a small number of countries that, as a matter of policy due to their size, geographic location, or specific perceived needs, appoint top-quality international facultysuch as Hong Kong, Singapore, and Switzerland. A third group teaches at universities in countries where there is a shortage of local staff-such as Saudi Arabia and other Gulf countries, some African countries, and a few others. Here, international academics are frequently hired to teach lower level courses, often come from Egypt, South Asia, or other regions, and frequently from nonprestigious universities. The fourth category, which overlaps with the first three, consists of diaspora academics that immigrated from 